\title{
The influence of plasma defocusing in high harmonic generation
}

\author{
Chien-Jen Lai ${ }^{1}$ and Franz X. Kärtner ${ }^{1,2, *}$ \\ ${ }^{1}$ Department of Electrical Engineering and Computer Science and Research Laboratory of Electronics, \\ Massachusetts Institute of Technology, 77 Massachusetts Avenue, Cambridge, MA 02139, USA \\ ${ }^{2}$ Center for Free-Electron Laser Science, DESY, and Department of Physics, University of Hamburg, 22607 \\ Hamburg, Germany \\ *kaertner@mit.edu
}

\begin{abstract}
We numerically investigate the influence of plasma defocusing in high harmonic generation (HHG) by solving the first-order wave equation in an ionized medium and defining an enhancement factor to quantitatively analyze the influence of plasma defocusing. While degrading the driver pulse intensity, plasma also has a strong impact on HHG phase-matching. Combined with the HHG wavelength scaling law, our results give an estimate of HHG efficiencies with different driver wavelengths and show a limited HHG efficiency in high density media.
\end{abstract}

(C)2011 Optical Society of America

OCIS codes: (020.2649) Strong field laser physics; (320.7110) Ultrafast nonlinear optics.

\section{References and links}

1. Ch. Spielmann, N. H. Burnett, S. Sartania, R. Koppitsch, M. Schnurer, C. Kan, M. Lenzner, P. Wobrauschek, and F. Krausz, "Generation of Coherent X-rays in the Water Window Using 5-Femtosecond Laser Pulses," Science 278(5338), 661-664 (1997).

2. J. Seres, E. Seres, A. J. Verhoef, G. Tempea, C. Streli, P. Wobrauschek, V. Yakovlev, A. Scrinzi, C. Spielmann, and F. Krausz, "Laser technology: source of coherent kiloelectronvolt X-rays," Nature 433(7026), 596-596 (2005).

3. M.-C. Chen, P. Arpin, T. Popmintchev, M. Gerrity, B. Zhang, M. Seaberg, D. Popmintchev, M. M. Murnane, and H. C. Kapteyn, "Bright, coherent, ultrafast soft X-ray harmonics spanning the water window from a tabletop light source," Phys. Rev. Lett. 105(17), 173901 (2010).

4. T. Popmintchev, M.-C. Chen, A. Bahabad, M. Gerrity, P. Sidorenko, O. Cohen, I. P. Christov, M. M. Murnane, and H. C. Kapteyn, "Phase matching of high harmonic generation in the soft and hard X-ray regions of the spectrum,” Proc. Natl. Acad. Sci. U.S.A. 106(26), 10516-10521 (2009).

5. J. Moses, S.-W. Huang, K.-H. Hong, O. D. Mucke, E. L. Falcao-Filho, A. Benedick, F. O. Ilday, A. Dergachev, J. A. Bolger, B. J. Eggleton, and F. X. Kartner, "Highly stable ultrabroadband mid-infrared optical parametric chirped pulse amplifier optimized for superfluorescence suppression,” Opt. Lett. 34(11), 1639-1641 (2009).

6. P. Colosimo, G. Doumy, C. I. Blaga, J. Wheeler, C. Hauri, F. Catoire, J. Tate, R. Chirla, A. M. March, G. G. Paulus, H. G. Muller, P. Agostini, and L. F. Dimauro, "Scaling strong-field interactions towards the classical limit," Nat. Phys. 4(5), 386-389 (2008).

7. E. J. Takahashi, T. Kanai, K. L. Ishikawa, Y. Nabekawa, and K. Midorikawa, "Coherent water window x ray by phase-matched high-order harmonic generation in neutral media," Phys. Rev. Lett. 101(25), 253901 (2008).

8. K.-H. Hong, J. T. Gopinath, D. Rand, A. M. Siddiqui, S.-W. Huang, E. Li, B. J. Eggleton, J. D. Hybl, T. Y. Fan, and F. X. Kartner, "High-energy, kHz-repetition-rate, ps cryogenic Yb:YAG chirped-pulse amplifier," Opt. Lett. 35(11), 1752-1754 (2010).

9. E. L. Falcao-Filho, M. Gkortsas, A. Gordon, and F. X. Kartner, "Analytic scaling analysis of high harmonic generation conversion efficiency," Opt. Express 17(13), 11217-11229 (2009).

10. J. Tate, T. Auguste, H. G. Muller, P. Salieres, P. Agostini, and L. F. DiMauro, "Scaling of wave-packet dynamics in an intense midinfrared field," Phys. Rev. Lett. 98(1), 013901 (2007).

11. A. D. Shiner, C. Trallero-Herrero, N. Kajumba, H.-C. Bandulet, D. Comtois, F. Legare, M. Giguere, J.-C. Kieffer, P. B. Corkum, and D. M. Villeneuve, "Wavelength scaling of high harmonic generation efficiency," Phys. Rev. Lett. 103(7), 073902 (2009).

12. C. Altucci, T. Starczewski, E. Mevel, C.-G. Wahlstrom, B. Carre, and A. L'Huillier, "Influence of atomic density in high-order harmonic generation," J. Opt. Soc. Am. B 13(1), 148-156 (1996).

13. I. Jong Kim, G. H. Lee, S. B. Park, Y. S. Lee, T. K. Kim, C. H. Nam, T. Mocek, and K. Jakubczak, "Generation of submicrojoule high harmonics using a long gas jet in a two-color laser field," Appl. Phys. Lett. 92, 021125 (2008).

14. H. Dachraoui, T. Auguste, A. Helmstedt, P. Bartz, M. Michelswirth, N. Mueller, W. Pfeiffer, P. Salieres, and U. Heinzmann, "Interplay between absorption, dispersion and refraction in high-order harmonic generation," J. Phys. At. Mol. Opt. Phys. 42(17), 175402 (2009). 
15. V. Tosa, E. Balogh, and K. Kovács, "Phase-matched generation of water-window x rays," Phys. Rev. A 80(4), 045801 (2009).

16. V. Tosa, K. T. Kim, and C. H. Nam, "Macroscopic generation of attosecond-pulse trains in strongly ionized media," Phys. Rev. A 79(4), 043828 (2009).

17. E. Constant, D. Garzella, P. Breger, E. Mevel, Ch. Dorrer, C. Le Blanc, F. Salin, and P. Agostini, "Optimizing High Harmonic Generation in Absorbing Gases: Model and Experiment," Phys. Rev. Lett. 82(8), 1668-1671 (1999).

18. M. Geissler, G. Tempea, A. Scrinzi, M. Schnurer, F. Krausz, and T. Brabec, "Light Propagation in Field-Ionizing Media: Extreme Nonlinear Optics," Phys. Rev. Lett. 83(15), 2930-2933 (1999).

19. M. V. Ammosov, N. B. Delone, and V. P. Krainov, "Tunnel ionization of complex atoms and of atomic ions in an alternating electromagnetic field," Sov. Phys. JETP 64(6), 1191-1194 (1986).

20. A. Behjat, G. J. Tallents, and D. Neely, "The characterization of a high-density gas jet," J. Phys. D Appl. Phys. 30(20), 2872-2879 (1997).

21. J.-S. Wu, S.-Y. Chou, U.-M. Lee, Y.-L. Shao, and Y.-Y. Lian, "Parallel DSMC Simulation of a Single UnderExpanded Free Orifice Jet From Transition to Near-Continuum Regime,” J. Fluids Eng. 127(6), 1161-1170 (2005).

22. E. J. Takahashi, Y. Nabekawa, H. Mashiko, H. Hasegawa, A. Suda, and K. Midorikawa, "Generation of Strong Optical Field in Soft X-ray Region by Using High-Order Harmonics,” IEEE J. Sel. Top. Quantum Electron. 10(6), 1315-1328 (2004)

23. S. Kazamias, S. Daboussi, O. Guilbaud, K. Cassou, D. Ros, B. Cros, and G. Maynard, "Pressure-induced phase matching in high-order harmonic generation," Phys. Rev. A 83(6), 063405 (2011).

24. Pascal Salières and Ivan Christov, "Macroscopic Effects in High-Order Harmonic Generation," in Strong Field Laser Physics, Thomas Brabec, ed. (Springer, 2008).

25. M. Lewenstein, P. Salières, and A. L'Huillier, "Phase of the atomic polarization in high-order harmonic generation," Phys. Rev. A 52(6), 4747-4754 (1995).

26. M. J. Weber, Handbook of Optical Materials (CRC Press, 2003), Chap. 6.

27. B. L. Henke, E. M. Gullikson, and J. C. Davis, "X-ray interactions: photoabsorption, scattering, transmision, and reflection at E=50-30000 eV, Z=1-92," At. Data Nucl. Data Tables 54(2), 181-342 (1993).

28. I. J. Kim, C. M. Kim, H. T. Kim, G. H. Lee, Y. S. Lee, J. Y. Park, D. J. Cho, and C. H. Nam, "Highly Efficient High-Harmonic Generation in an Orthogonally Polarized Two-Color Laser Field," Phys. Rev. Lett. 94, 243901 (2005).

29. E. J. Takahashi, Y. Nabekawa, and K. Midorikawa, "Low-divergence coherent soft X-ray source at $13 \mathrm{~nm}$ by high-order harmonics," Appl. Phys. Lett. 84(1), 4-6 (2004).

\section{Introduction}

High harmonic generation (HHG) has become a promising way towards table-top coherent EUV and soft X-ray light sources [1-3]. In recent years, it has been shown theoretically and experimentally, that the maximum available photon energy from HHG based sources can be extended to the water window, and even $\mathrm{keV}$ photons are achievable by using longer driver wavelengths [4]. Therefore, techniques, such as optical parametric amplification (OPA), have been developed to generate high intensity IR pulses with wavelengths longer than the conventional 800nm wavelength from Ti:Sapphire amplifiers [5]. Several groups have already demonstrated HHG results with different IR driver pulses [3,6,7] and keep pushing OPA systems to higher pulse energy and longer wavelengths in pursuit of more efficient HHG and higher photon energy [8].

However, both theory and experiment show a severe drop of the single atom efficiency (SAE) of HHG that is proportional to $\lambda_{1}{ }^{-(5 \sim 6)}$, at a given HHG driver wavelength $\lambda_{1}$ [9-11]. To compensate the low single atom efficiency, one straight forward way is to increase the number of participating atoms by applying higher pressure to the interaction region or equivalently extending its length. This approach especially improves the low HHG efficiency of helium [9]. For low atomic density, the efficiency is enhanced quadratically with pressure [12], but plasma defocusing starts to become important as the free electron density increases. A previous study has shown that plasma defocusing in neon at $80 \mathrm{mbar}$ can significantly reduce the peak intensity of an $800 \mathrm{~nm}$ driver pulse and diverges the driver pulse energy within a few hundred microns [12]. This effect not only decreases the cutoff photon energy but also shortens the interaction length for HHG. Although many HHG works have emphasized the importance of plasma defocusing and attributed many experimental phenomena to it [12-16], a quantitative discussion about the influence of plasma defocusing in HHG efficiency is missing.

\#153148 - \$15.00 USD

(C) 2011 OSA
Received 19 Aug 2011; revised 3 Oct 2011; accepted 8 Oct 2011; published 24 Oct 2011 7 November 2011 / Vol. 19, No. 23 / OPTICS EXPRESS 22378 
In this work, we numerically investigate plasma defocusing on IR pulse propagation in $\mathrm{Ne}$ and $\mathrm{He}$ and discuss how it affects HHG by defining an enhancement factor, which is a generalization of the analysis of Ref [17]. For different pressure, the pulse intensity and ionization level may then show quite different distributions that strongly impact the phasematching condition and HHG efficiency. The enhancement factor shows how HHG efficiency varies when the medium pressure changes or different gases and medium geometries are used. According to our result, plasma defocusing has strong impact on both the phase-matching condition and the driver pulse intensity. Increasing medium pressure only results in a limited enhancement of the HHG efficiency even if reabsorption is negligible, and it can only partially compensate the loss of SAE when using longer wavelength driver pulses.

\section{Numerical model for plasma defocusing}

Our numerical analysis first calculates how an optical pulse propagates and interacts with HHG gas media. In our situation, the model equation should include the effects of diffraction, self-focusing, plasma defocusing, and ionization loss. Dispersion and other nonlinear effects can be neglected because the medium under consideration is either a short gas jet or a lowpressure $1 \mathrm{~cm}$-long gas cell. In the slowly evolving wave approximation, a first order propagation equation adapted from Ref [18]. is employed in our model:

$$
\frac{\partial E}{\partial z}=\frac{i}{2 k} \nabla_{\perp}^{2} E+i \frac{k}{2} n_{2} \varepsilon_{0} c|E|^{2} E-\frac{1}{2 c} \int_{-\infty}^{\tau} \omega_{p}^{2} E d \tau^{\prime}-\frac{I_{p}}{2 c \varepsilon_{0} \operatorname{Re}(E)^{2}} \frac{\partial \rho}{\partial \tau} E,
$$

where, $E$ is the complex representation of the electric field; $z$ and $\tau$ are the propagation distance and the retarded time in the retarded time frame respectively; $k$ is the wave-vector at the carrier frequency; $\nabla_{\perp}^{2}$ is the transversal Laplace operator; $n_{2}$ is the nonlinear index of refraction; $\omega_{p}$ is the plasma frequency; $I_{p}$ is the ionization potential of the atom; $\rho$ is the number density of the ionized atoms. We assume that tunneling ionization is the only ionization mechanism, and therefore, the ADK formula gives an accurate description of the ionization rate $\partial \rho / \partial \tau$ [19]. Since tunneling ionization depends on the phase of the electric field, the ionization loss term contains the real part of the electric field. Because of cylindrical symmetry, $E$ is a function of $z, \tau$, and the radial coordinate $r$.

In our numerical study, we consider two different gases interact with a $2.5 \mathrm{~mJ}, 40 \mathrm{fs}$ IR pulse centered at several different wavelengths. The gas is neon or helium because of their large ionization potentials and high HHG cutoffs. The geometry of the gas nozzle is either a gas jet or a gas cell both of which are frequently used in experiment. The gas jet is assumed to have a Gaussian pressure distribution with $1 \mathrm{~mm}$ full-width-half-maximum (FWHM) [20], and the laser pulse is focused at the center of the gas jet. The gas cell length is $1 \mathrm{~cm}$, and the laser pulse is focused at the center of the cell, too. The pressure profile of the gas cell is assumed to be uniform inside the cell and has a Lorentzian shape at both ends [21]. Although it has been shown experimentally that the relative position between the focus and the medium can be optimized for efficiency, we neglect this tuning degree of freedom for simplicity. We consider six different wavelengths $0.8 \mu \mathrm{m}, 1.3 \mu \mathrm{m}, 1.6 \mu \mathrm{m}, 2 \mu \mathrm{m}, 3 \mu \mathrm{m}$, and $4 \mu \mathrm{m}$, which cover most wavelengths of interest in current near and mid-IR HHG experiments. For comparison, all IR pulses have the same pulse energy $2.5 \mathrm{~mJ}$ and pulse duration $40 \mathrm{fs}$. The beam sizes are $75 \mu \mathrm{m}$ in $\mathrm{He}$ and $95 \mu \mathrm{m}$ in Ne that correspond to intensities of $7 \times 10^{14} \mathrm{~W} / \mathrm{cm}^{2}$ and $4.4 \times 10^{14} \mathrm{~W} / \mathrm{cm}^{2}$ respectively. In this paper, the medium temperature is assumed to be $300 \mathrm{~K}$, and then $1 \mathrm{bar}$ pressure is equivalent to a density of $2.4 \times 10^{19}$ atoms $/ \mathrm{cm}^{3}$ if we assume the medium is an ideal gas. In a real gas jet where the temperature can be much lower than $300 \mathrm{~K}$ due to free expansion, the pressure in our simulation should be referred as atomic density.

Figure 1 shows the peak intensity and ionization level distribution when a $2.5 \mathrm{~mJ}, 40 \mathrm{fs}$, $2 \mu \mathrm{m}$ laser pulse propagates from negative $z$ to positive $z$ through a He jet with three different pressures. The laser pulse is focused to an intensity of $7 \times 10^{14} \mathrm{~W} / \mathrm{cm}^{2}$ at the center of the jets $(z$ $=0$ ). Figure 1 (a)-(c) show the peak intensity of the pulse during propagation. The jet is 
located at $z=0$, and the Gaussian curve in each plot shows a relative He pressure (atomic density) profile along the $\mathrm{z}$-dimension. Because the beam spot is much smaller than the characteristic length of the jet, we neglect the pressure variation in the transverse spatial dimensions. In Fig. 1(a), the gas density is small, so the plasma doesn't have too much impact on the pulse, and the intensity evolution is similar to free space propagation. As the pressure becomes higher in Fig. 1 (b) and (c), plasma defocusing starts to play an important role. A drop of intensity near the center of the jet can be seen. Such a drop of intensity hinders the pulse from efficiently ionizing the gas and generating high harmonics. Figure 1 (d)-(f) show the distribution of the ionization level (i.e. the fraction of atoms that are ionized) after the entire pulse leaves the medium and before the plasma starts to diffuse or recombine with ions. The pressures in Fig. 1 (d)-(f) are the same as in Fig. 1 (a)-(c) respectively. Because ionization is a highly nonlinear process, the ionization level is more sensitive to plasma defocusing than the peak intensity of the laser field. While the ionization level in Fig. 1(d) distributes widely from negative to positive $z$ domain, the ionized regions in Fig. 1 (e) and (f) are mostly restricted in the negative $\mathrm{z}$ domain. Furthermore, the strong defocusing effect in Fig. 1(f) even prevents the pulse from approaching the center of the gas jet with a high intensity, so the ionization and HHG can only occur in the low pressure wing before the gas jet. In such situation, the high density center of the gas jet doesn't help HHG but reabsorbs the high harmonic photons instead. This effect decreases the advantage of using a high pressure gas jet.
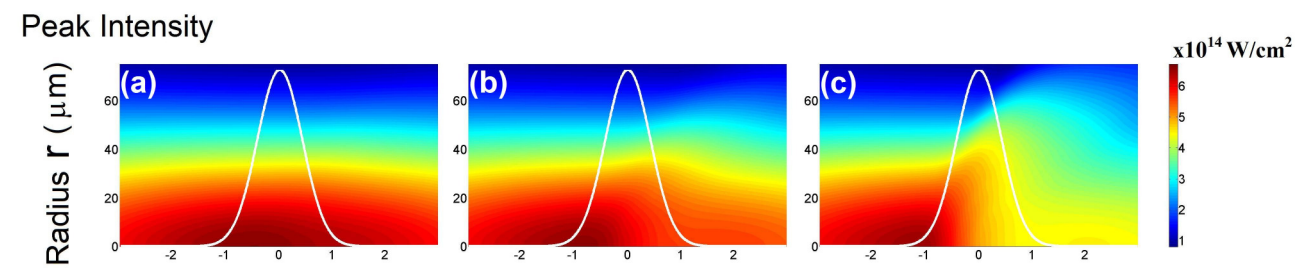

\section{Ionization Level}
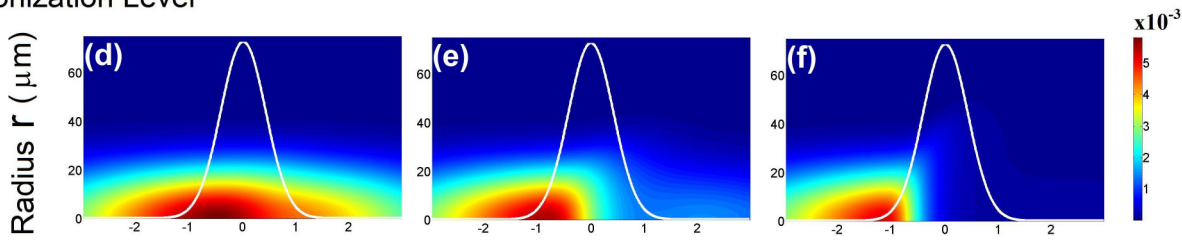

Propagation Distance Z (mm)

Fig. 1. Numerical results of the $2 \mu \mathrm{m}$ laser pulse propagation toward the $+z$ direction through a He gas jet. The upper row shows the peak intensity when the peak pressure at the jet is (a) $0.1 \mathrm{bar}$, (b) $1 \mathrm{bar}$, and (c) 10bar. The Gaussian curves illustrate the relative pressure profile of the jet. The lower row shows the ionization level when the pressure is (d) $0.1 \mathrm{bar}$, (e) 1bar, and (f) 10bar. The color scales of the intensity and the ionization level are shown on the side.

Figure 2 shows the peak intensity and ionization level distributions when the same pulse considered in Fig. 1 propagates through a 1 $\mathrm{cm}$-long helium cell with different pressures. The laser pulse is focused to the same intensity $\left(7 \times 10^{14} \mathrm{~W} / \mathrm{cm}^{2}\right)$ at the center of the cell $(z=0)$. Compared with the jet case, the intensity and ionization level in the He cell are lower at the same pressure because the pulse has to propagate a longer distance in the cell and suffers more plasma defocusing before reaching the focus at the cell center. The ionization level again shows sensitive dependence on the laser pulse intensity. In Fig. 2(f), the maximum ionization is only $4 \times 10^{-4}$, which is much less than the ionization level in the lower pressure case (Fig. 2(d)). An immediate effect of plasma defocusing can be seen from Fig. 1 and Fig. 2 is the drop of the pulse intensity that reduces the cutoff photon energy or even stops HHG. 
Besides, as will be shown in next section, the impact of plasma defocusing on the phasematching condition is even more dramatic to HHG efficiency.

Peak Intensity
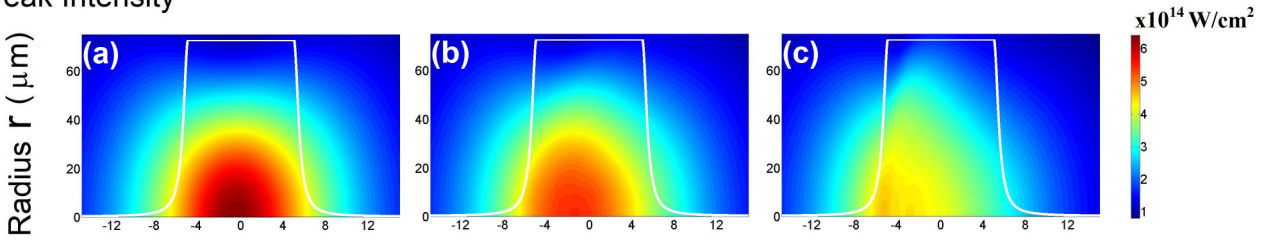

\section{Ionization Level}
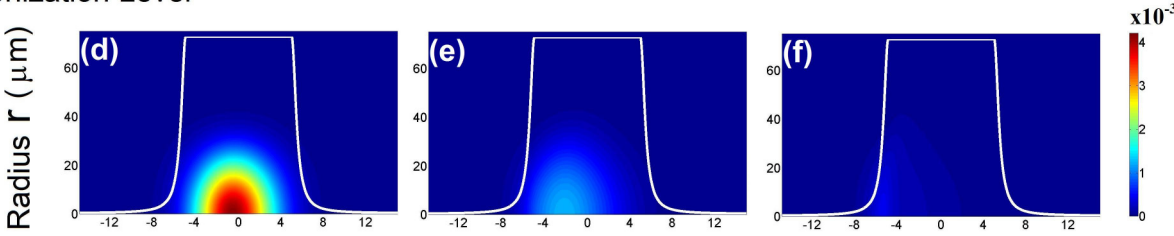

Propagation Distance $\mathbf{Z}(\mathrm{mm})$

Fig. 2. Numerical results of the $2 \mu \mathrm{m}$ laser pulse propagation toward the $+z$ direction through a He cell. The upper row shows the peak intensity when the pressure is (a) 0.1 bar, (b) 1 bar, and (c) 10bar. The relative pressure is shown by the flat-top curve. The lower row shows the ionization level when the pressure is (d) 0.1bar, (e) 1bar, and (f) 10bar. The color scales of the intensity and the ionization level are shown on the side.

\section{Phase-matching and HHG enhancement}

To quantitatively discuss the influence of plasma defocusing, we introduce and calculate the HHG efficiency for the various cases. However, solving a three dimensional (3D) timedependent Schrodinger equation along with the 3D wave propagation is time-consuming, so we follow the analysis of Ref [17]. and generalize it to include plasma defocusing effect. It captures and analyzes the macroscopic properties of the HHG process, including phasematching and reabsorption of the harmonics, and shows excellent agreement with experiment and HHG energy scaling [22,23]. The HHG energy of the $q^{\text {th }}$ harmonic is assumed to be in the following form:

$$
\begin{aligned}
E_{q} & =A \eta\left(q, \lambda_{1}\right) \int 2 \pi r d r \int d \tau \times \\
& \left|\int_{-L / 2}^{L / 2} \sqrt{w} P(z) \exp \left[i z \Delta k_{q}(r, z, \tau)\right] \exp [-\beta(z)(L-z)] d z\right|^{2},
\end{aligned}
$$

where $A$ is a proportional constant; $\eta\left(q, \lambda_{1}\right)$, as a function of the harmonic order $q$ and the driver wavelength $\lambda_{l}$, is the single atom efficiency (SAE); $w=w(r, z, \tau)$ is the ionization rate given by the ADK formula; $P(z)$ is the medium pressure; $\Delta k_{q}=q k_{1}-k_{q}$ is the mismatch between the fundamental and the $q^{\text {th }}$ harmonic wave-vectors; $\beta(z)$ represents the absorption of the harmonics. The integral with respect to $z$ is due to the coherent addition of the high harmonic field generated over the non-uniform pressure distribution $P(z)$. The integral is over a range from $-L / 2$ to $L / 2$ that is long enough to cover the medium completely. Its magnitude square would be proportional to the harmonic intensity generated from the point $(r, \tau)$ in the parameter space. Then, the integrals over $r$ and $\tau$ consider the HHG contribution from every part of the driver pulse and result in the total amount of high-harmonic energy in a given harmonic. We neglect the depletion of the ground states because the ionization level considered here is always less than few percents. We define the integral part of Eq. (2) as the enhancement factor $\xi$ : 


$$
\xi \equiv \int 2 \pi r d r \int d \tau\left|\int_{-L / 2}^{L / 2} \sqrt{w} P(z) \exp \left[i z \Delta k_{q}(r, z, \tau)\right] \exp [-\beta(L-z)] d z\right|^{2},
$$

because it shows how much the SAE is enhanced by the total HHG medium. Then the total high harmonic energy can be rewritten as

$$
E_{q}=A \eta \xi
$$

This enhancement factor $\xi$ comprises the propagation effects of $\mathrm{HHG}$ and is proportional to the high harmonic energy, so it is useful for the investigation of the plasma defocusing effect.

The wave-vector mismatch $\Delta k_{q}$ is important in the calculation of the enhancement factor $\xi$. For a many-cycle pulse, phase-matching mainly depends on the neutral atom dispersion $\delta k_{a}$, the plasma dispersion $\delta k_{p}$ that is induced by the free electrons, and the mismatches $\delta k_{g}$ and $\delta k_{d}$ that result from the geometric phase and the dipole phase respectively [14,24]. Therefore, the total wave-vector mismatch can be expressed as

$$
\Delta k_{q}=\delta k_{a}+\delta k_{p}+\delta k_{g}+\delta k_{d}
$$

The geometric and dipole phases are particularly important for longer driver wavelengths for several reasons. Because most mid-IR OPA or OPCPA systems have to be focused tightly (usually with a beam size smaller than $100 \mu \mathrm{m}$ ) to generate high harmonics, and then, the Guoy and dipole phases are not negligible. Besides, the Guoy phase is proportional to the driver wavelength for a fixed spot size, and the dipole phase is proportional to the driver wavelength cube due to the longer trajectory and higher kinetic energy of electron during propagation. Last, the wave-vector mismatch is also proportional to the harmonic order that is larger for longer driver wavelength. Here we consider the contribution from the shorttrajectory to HHG, and the dipole phase can be written as $-\alpha_{s} I$, where $I$ is the laser intensity, and the coefficient $\alpha_{s}$ can be calculated based on the harmonic order and the action of the semi-classical electron trajectory [25]. In the following simulation, $\alpha_{s}$ is $5 \times 10^{-14} \mathrm{rad}-\mathrm{cm}^{2} / \mathrm{W}$ for $0.8 \mu \mathrm{m}$ driver wavelength. For other driver wavelengths, the coefficients are scaled with the cube of the wavelength.
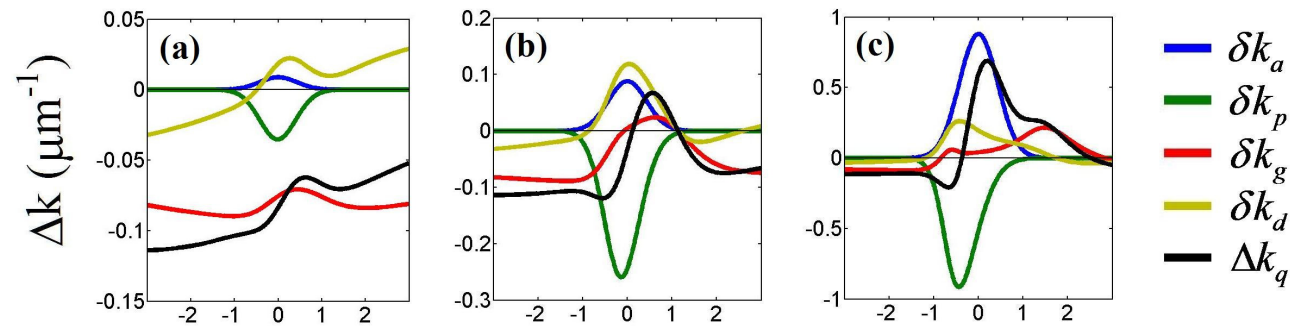

\section{Propagation Distance $\mathbf{Z}(\mathrm{mm})$}

Fig. 3. The wave-vector mismatches in a He jet at $r=0$ and $\tau=0$ (pulse peak). The laser pulse and medium conditions are the same as Fig. 1, and the medium pressures are (a) 0.1bar, (b) 1 bar, and (c) 10bar respectively. The harmonic energy considered here is $500 \mathrm{eV}$. In each plot, the blue, green, red, and yellow curves show the mismatch due to neutral atom dispersion, plasma dispersion, geometric phase, and dipole phase respectively. The black curve shows the total wave-vector mismatch that is the sum of the above four curves. 

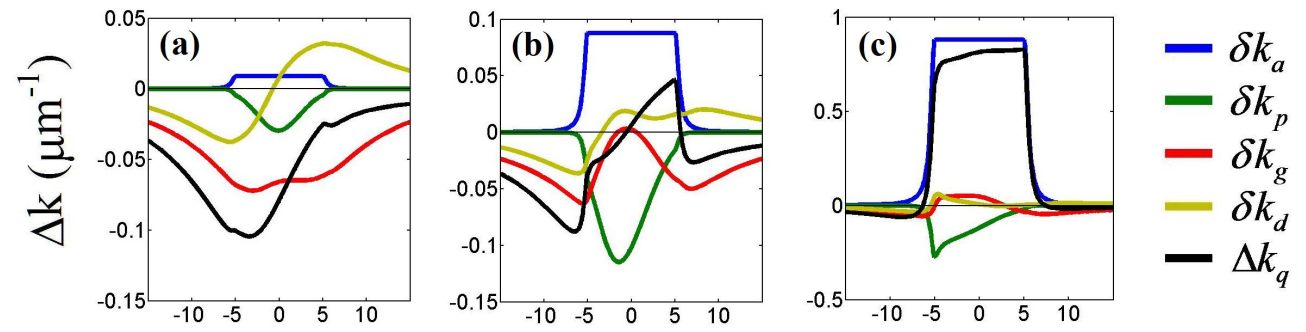

\section{Propagation Distance $\mathbf{Z}(\mathrm{mm})$}

Fig. 4. The wave-vector mismatches in a He cell at $r=0$ and $\tau=0$ (pulse peak). The laser pulse and medium conditions are the same as Fig. 2, and the medium pressure is (a) $0.1 \mathrm{bar}$, (b) $1 \mathrm{bar}$, and (c) $10 \mathrm{bar}$ respectively. The harmonic energy considered here is $500 \mathrm{eV}$. In each plot, the blue, green, red, and yellow curves show the mismatch due to neutral atom dispersion, plasma dispersion, geometric phase, and dipole phase respectively. The black curve shows the total wave-vector mismatch that is the sum of the above four curves.

Figure 3 and Fig. 4 show and compare the four different sources of wave-vector mismatch seen by the peak of the driver pulse (i.e. at $r=0$ and $\tau=0$ ) under the same driver pulse condition and medium structure as Fig. 1 and Fig. 2 respectively. The harmonic energy considered in both figures is $500 \mathrm{eV}$, which is the $807 \mathrm{th}$ harmonic of the $2 \mu \mathrm{m}$ driver wavelength. The refractive indices used here for the driver pulses and the harmonics are taken from Ref [26]. and Ref [27]. respectively. At low pressure (Fig. 3(a) and Fig. 4(a), $P=$ $0.1 \mathrm{bar}$ ), the geometric phase and the dipole phase are more dominant than the other two, and phase-matching $\left(\Delta k_{q}=0\right)$ can only be realized far behind the focus. As the pressure becomes higher (Fig. 3(b) and Fig. 4(b), $P=1$ bar), all four contributions are comparable, and the total $\Delta k_{q}$ shows complicated behavior. Phase-matching is achieved at some point near the center of the medium. At even higher pressure (Fig. 3(c) and Fig. 4(c), $P=10 \mathrm{bar}$ ), the total $\Delta k_{q}$ basically follows the profile of the neutral atom dispersion because the negative plasma dispersion is roughly cancelled by the positive dipole and geometric phases.

When the ionization level is maintained below a few percent, the neutral atom dispersion is not affected by the plasma, but the other three phase-matching factors are still significantly influenced by plasma defocusing. As shown in Fig. 1 and Fig. 2, the ionization level that determines the plasma dispersion is very sensitive to the driver pulse intensity, which drops faster due to plasma defocusing. The fast drop of the driver pulse intensity then results in an enhanced dipole phase because the dipole phase of the short trajectory is proportional to the negative derivative of the driver pulse intensity [24]. The geometric phase also changes due to the modification of the intensity profile along the radius direction. At low pressure, the geometric phase is almost the same as the Guoy phase of a focused Gaussian beam. When the pressure increases, and the plasma defocusing becomes stronger, the geometric phase that results from the diffraction can be quite different from the Guoy phase as shown in Fig. 3 and Fig. 4. Therefore, plasma defocusing not only reduces the pulse intensity, but also influences the phase-matching. It is difficult to control and balance all these factors around $\Delta k_{q}=0$ for a long distance. Instead, $\Delta k_{q}$ often crosses the $\Delta k_{q}=0$ line with some slope. To improve the HHG efficiency in such a situation, one should try to decrease the slope and move the crossing point to the high density part of the medium. 

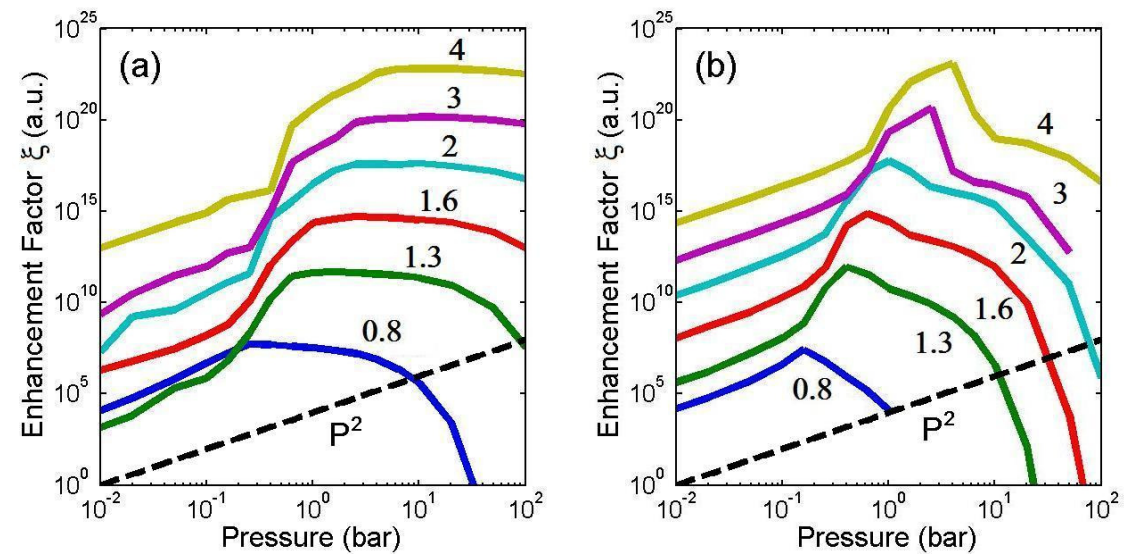

Fig. 5. Enhancement factor $\xi$ for He. (a) and (b) show the enhancement of a $1 \mathrm{~mm} \mathrm{He} \mathrm{jet} \mathrm{and} \mathrm{a}$ $1 \mathrm{~cm}$ He cell respectively. For each driver wavelength, the curve is intentionally shifted for clarity. The number near each curve indicates the driver wavelength in units of $1 \mu \mathrm{m}$. For comparison, the dashed lines show a parabolic dependence on pressure.
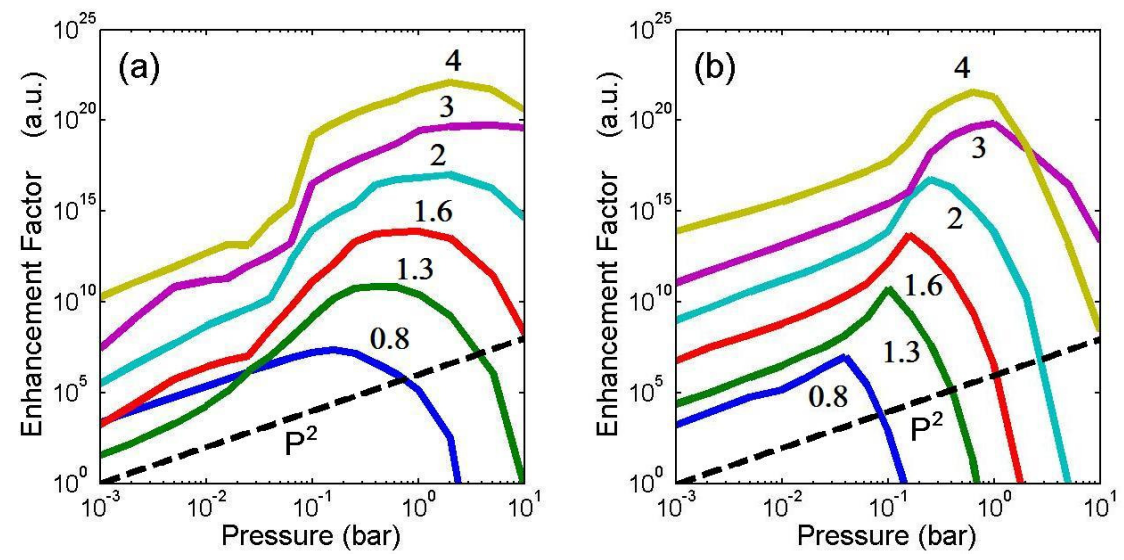

Fig. 6. Enhancement factor $\xi$ for Ne. (a) and (b) show the enhancement of a $1 \mathrm{~mm} \mathrm{Ne} \mathrm{jet} \mathrm{and} \mathrm{a}$ $1 \mathrm{~cm} \mathrm{Ne}$ cell respectively. For each driver wavelength, the curve is intentionally shifted for clarity. The number near each curve indicates the driver wavelength in units of $1 \mu \mathrm{m}$. For comparison, the dashed lines show a parabolic dependence on pressure.

Figure 5 shows the calculated enhancement factors $\xi$ for a He jet and a He cell for six different driver wavelengths and varying pressure. In Fig. 5(a), there are sharp transitions at about $0.1 \mathrm{bar}$, because at this pressure a transition from phase-mismatched HHG to phasematched HHG occurs. At low pressure $(<0.1 \mathrm{bar})$, Guoy phase and dipole phase dominate, and the phase-matching point where $\Delta k_{q}=0$ is located far behind the gas jet. Therefore, phasematching cannot be achieved with low density media density, and poor HHG efficiency results from it. When the pressure is low, $\Delta k_{q}$ doesn't change much with the increasing pressure. Therefore the HHG efficiency still increases quadratically with the medium pressure although it is not phase-matched at all. Once the pressure is high enough $(>0.1 \mathrm{bar})$, the neutral atom dispersion is able to compensate the other phase effects and results in a phase-matching point within the gas jet. Correspondingly the enhancement factor $\xi$, and with it the HHG efficiency, increase drastically. This transition phenomenon is less sharp for shorter driver wavelengths due to the smaller Guoy and dipole phases and smaller harmonic orders that can be generated. With higher medium density, the strong plasma defocusing and reabsorption start to limit the HHG efficiency and result in a saturation feature with optimal medium 
pressure for $\xi$. In Fig. 5(b), the basic features of a He cell are similar to those of a He jet except that the optimal pressure is lower due to a longer interaction length. Figure 6 shows the enhancement factor $\xi$ for Ne. The same sharp transition can be seen at lower medium pressures because $\mathrm{Ne}$ has larger neutral atom dispersion. Compared to $\mathrm{He}$, the larger reabsorption of $\mathrm{Ne}$ limits the optimal pressure to lower values than those of $\mathrm{He}$.

The calculated optimal pressures in Fig. 5 and Fig. 6 show excellent agreement with experiment. For $0.8 \mu \mathrm{m}$ driver wavelength, Fig. 5 and Fig. 6 show optimal HHG efficiency at a few hundreds mbar for gas jets and a few tens mbar for Ne cells. These are typical values appearing in experiment $[12,28]$. To further verify our model, we simulate the cases studied experimentally in Ref [22]. As shown in Fig. 7, the calculated optimal pressures for He and $\mathrm{Ne}$ are $25 \mathrm{mbar}$ and $18 \mathrm{mbar}$ respectively, which are close to the experimental pressures at $26 \mathrm{mbar}$ and $13 \mathrm{mbar}$. To show that these optimal pressures are closely related to plasma defocusing, we also calculate the enhancement factor without any plasma defocusing, as shown by the dashed curves in Fig. 7. The dashed curves are calculated by neglecting the impact of refractive index and losses due to plasma onto the driver pulses. The intensity distribution and the ionization level provide the information to calculate the enhancement factor, as shown in Fig. 7. The sharp turn on the dashed curve in Fig. 7(b) is due to phase mismatching. The initial intensity is too high and generates too much plasma that causes phase-mismatching. This phase-mismatched HHG has a coherence length [17] comparable to the medium length when the pressure is $10 \mathrm{mbar}$. When the pressure is higher than $10 \mathrm{mbar}$, the coherence length becomes shorter, and the enhancement cannot benefit more from the higher pressure. In Fig. 7(b), plasma defocusing decreases the intensity to an appropriate level for phase-matching, so the enhancement can be larger than for the case without plasmadefocusing. Beyond $0.8 \mu \mathrm{m}$ HHG experiments, our $1.6 \mu \mathrm{m}$ result also matches Ref [7]. well. The Ne jet in Ref. [7] is optimized at 0.76bar that is close to our optimal pressure at $1 \mathrm{bar}$ for a $\mathrm{Ne}$ jet. Determining the working pressure is important to the design of the vacuum system and the choice of appropriate gas geometry. The numerical analysis on plasma defocusing provided here gives the necessary information as well as the maximum enhancement due to the macroscopic propagation effects of the medium, and is therefore of great practical importance.
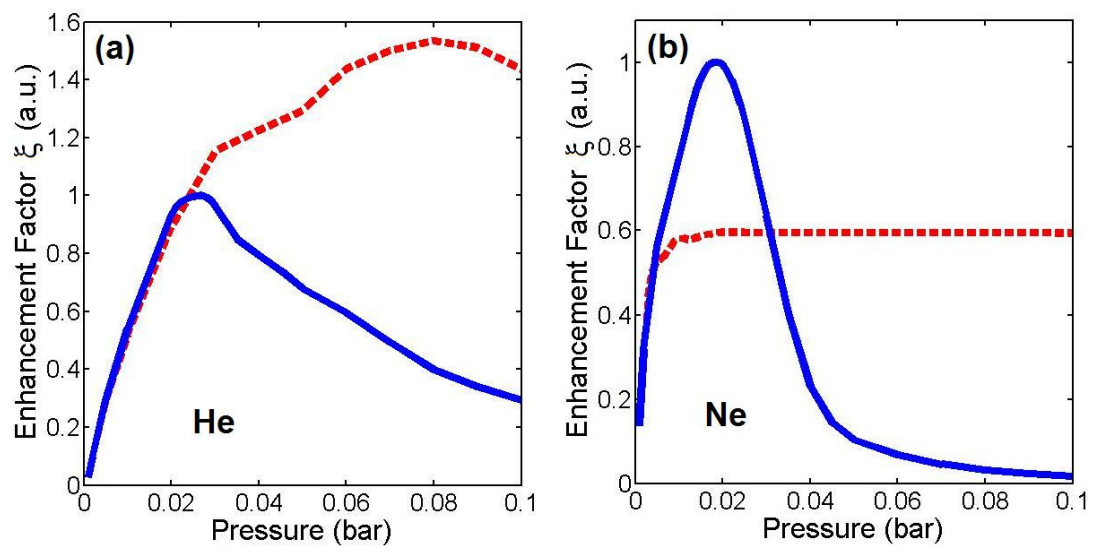

Fig. 7. Enhancement factor for $0.8 \mu \mathrm{m}$ driver wavelength with high pulse energy and loose focusing. For (a) He, a $130 \mathrm{~mJ}$ pulse is focused into a $5 \mathrm{~cm}$-long gas cell with $590 \mu \mathrm{m}$ beam radius. For (b) Ne, a $50 \mathrm{~mJ}$ pulse is focused into a $4 \mathrm{~cm}$-long gas cell with $360 \mu \mathrm{m}$ beam radius [22]. The solid curves consider plasma defocusing, while the dashed ones don't.

\section{HHG efficiency}

Although HHG efficiency is closely related to the microscopic dynamics of atoms under a strong field, our model can still estimate the efficiency for different driver wavelengths. 
According to Eq. (4), the enhancement factor $\xi$, the $\operatorname{SAE} \eta$, and the proportionality constant $A$ are needed to calculate the HHG efficiency. As shown in last section, the enhancement factor $\xi$ can be calculated by Eq. (3). Although the SAE $\eta$ is not discussed in this paper, it has been shown to scale with the driver wavelength as $\lambda_{1}{ }^{-(5 \sim 6)}$, and we can take $\eta=C \lambda_{1}{ }^{-5.5}$. The differences between SAE in the plateau are ignored. The unknown pre-factor $A \times C$ can be determined by comparing the theoretical scaling according to Eq. (2) with known experimental results, for instance, with an HHG experiment using a $0.8 \mu \mathrm{m}$ driver wavelength. This method is then able to give an estimate for the HHG efficiency with a different driver wavelength.
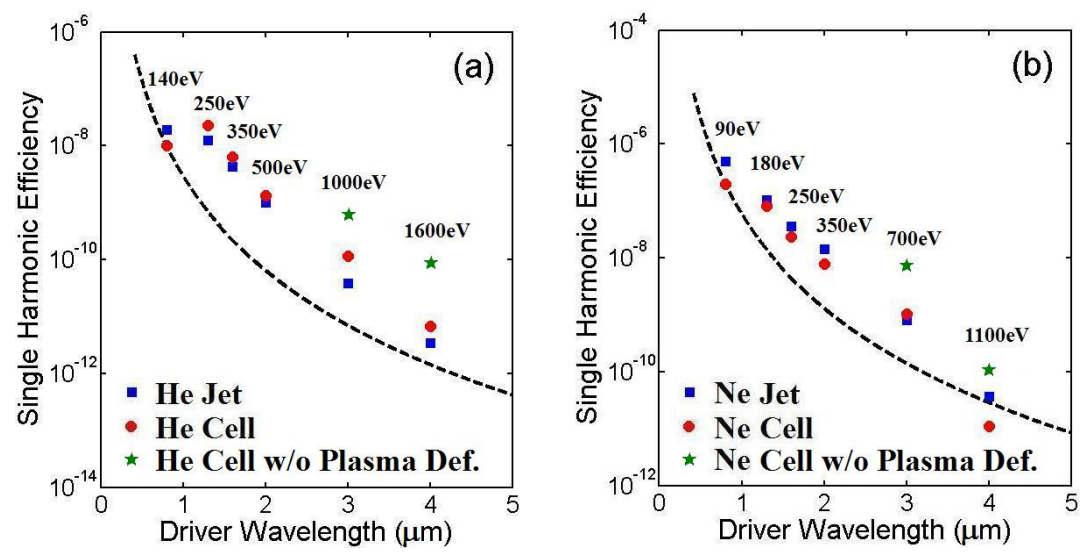

Fig. 8. Calculated HHG efficiency for optimal pressures in (a) He and (b) Ne. The black dotted curves also show a $\lambda_{1}{ }^{-5.5}$ dependence starting from the efficiency reference point. For different driver wavelengths, different photon energies that are near the corresponding cutoffs are considered, as labeled.

Figure 8(a) and (b) show the calculated HHG efficiency of He and Ne respectively. The reference efficiencies for $\mathrm{He}$ and $\mathrm{Ne} 0.8 \mu \mathrm{m}$ HHG are $1 \times 10^{-8}$ [22] and $2 \times 10^{-7}$ [29] respectively. Because the main advantage of using a longer driver wavelength is to generate higher cutoff, the efficiencies for different driver wavelengths are calculated at the photon energies near the cutoff of the HHG spectra. Our calculation shows similar results as the $1.6 \mu \mathrm{m}$ and $2 \mu \mathrm{m}$ experiments $[3,7]$. The dashed curves show a $\lambda_{1}{ }^{-5.5}$-scaling starting from the $0.8 \mu \mathrm{m}$ reference points. For comparison, we also calculate the cases without any plasma defocusing and show the results by the green stars. Most points are $0 \sim 2$ orders higher than the dashed curves, so it means that they have a few to hundreds times higher enhancements than the $0.8 \mu \mathrm{m}$ case. This only partially compensates the efficiency loss due to reduced SAE.

\section{Summary}

We quantitatively analyzed the influence of plasma defocusing on HHG for different driver wavelengths by defining and comparing the enhancement factor $\xi$ that considers macroscopic characteristics including plasma defocusing, reabsorption of harmonics, and phase-matching. Geometric and dipole phases that are important in the phase-matching of HHG driven by midIR wavelengths are also included. Our numerical result shows good agreement with experiment and provides an easy way to calculate and explain HHG performance without referring to the complex microscopic behavior of strong field dynamics and the atomic parameters. Although increasing the medium pressure can partially make up the severe loss of SAE with longer driver wavelengths, the compensation is still limited by the plasma defocusing. 


\section{Acknowledgments}

The authors acknowledge support by AFOSR Grants FA9550-09-1-0212, FA9550-10-1-0063 and by the Center for Free-Electron Laser Science at DESY, Hamburg.

\#153148 - \$15.00 USD (C) 2011 OSA
Received 19 Aug 2011; revised 3 Oct 2011; accepted 8 Oct 2011; published 24 Oct 2011 7 November 2011 / Vol. 19, No. 23 / OPTICS EXPRESS 22387 